\title{
АЛГОРИТМ ВЫБОРА ОПТИМАЛЬНОЙ СИНХРОНИЗАЦИИ ПРОЦЕССОВ В РАСПРЕДЕЛЕННОЙ ИМИТАЦИОННОЙ СИСТЕМЕ
}

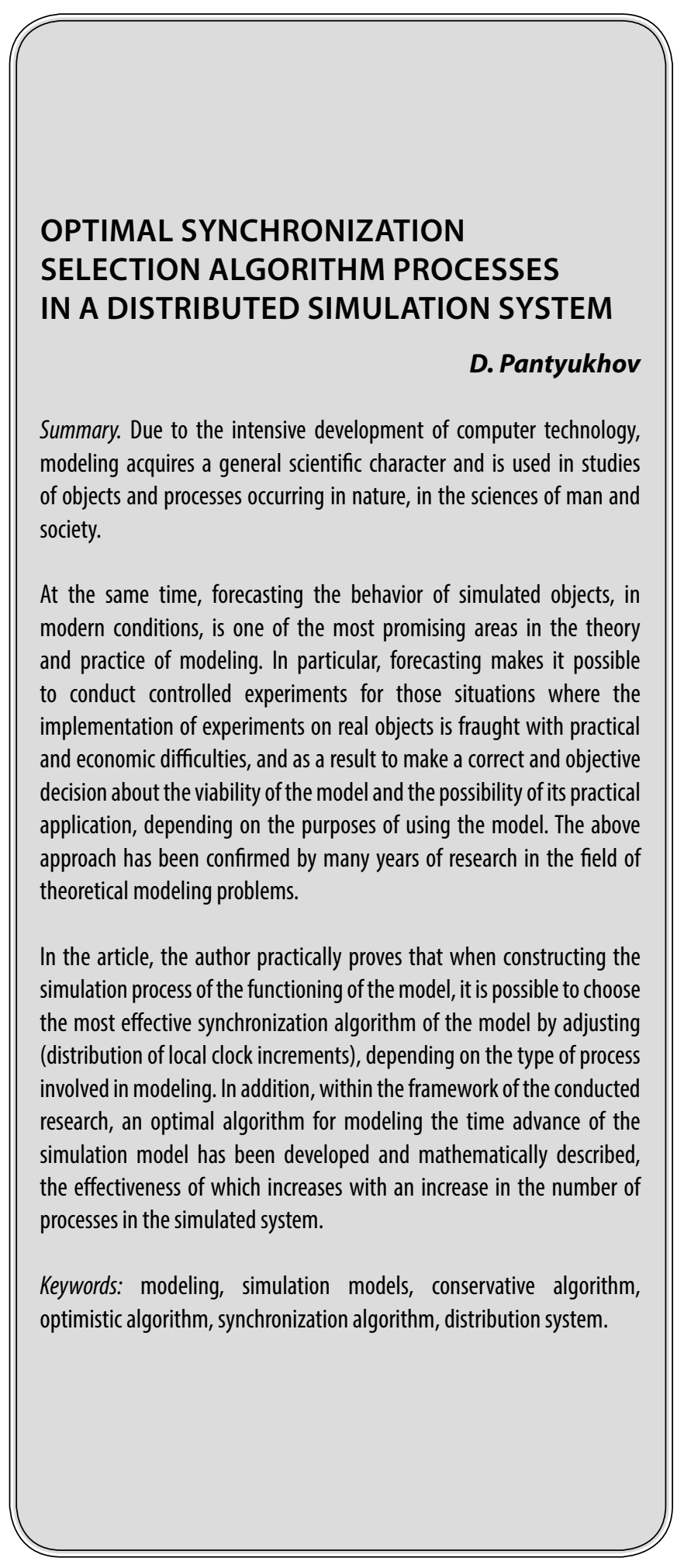

Пантюхов Дмитрий Валерьевич

Академия федеральной службы охраны Российской Федерации, г. Орёл gospamme@ya.ru

Аннотация. Благодаря интенсивному развитию вычислительной техники моделирование приобретает общенаучный характер и применяется в исследованиях объектов и процессов, происходящих в природе, а также в науках о человеке и обществе.

При этом прогнозирование поведения моделируемых объектов в современных условиях является одним из наиболее перспективных направлений в теории и практике моделирования. В частности, прогнозирование позволяет проводить контролируемые эксперименты для тех ситуаций, где осуществление экспериментов на реальных объектах сопряжено с трудностями практического и экономического характера, и в итоге принять правильное и объективное решение 0 жизнеспособности модели и возможности ее практического применения в зависимости от целей ее использования. Вышеприведенный подход подтвержден многолетними исследованиями в области теоретических проблем моделирования.

В статье автором практически доказывается, что при построении имитационного процесса функционирования модели можно путем корректировки (распределения приращений локальных часов) выбирать наиболее эффективный алгоритм синхронизации модели в зависимости от типа процесса, участвующего в моделировании. Кроме того, в рамках проведенного исследования разработан и математически описан оптимальный алгоритм моделирования продвижения времени имитационной модели, эффективность которого возрастает с увеличением числа процессов в моделируемой системе.

Ключевые слова: моделирование, имитационные модели, консервативный алгоритм, оптимистичный алгоритм, алгоритм синхронизации, распределительная система. 
$\mathbf{H}$ а протяжении последних сорока лет в научных исследованиях, посвященных моделированию различных объектов, активно обсуждался вопрос о том, как оптимизировать процессы моделирования для целей практического внедрения моделей в реальные условия [1, 3, 4, 8]. Одним из наиболее важных направлений оптимизации процессов моделирования стало прогнозирование поведения моделируемых объектов до их практического внедрения. Такой подход, как отмечается в специальной литературе [7, 8, 9, 13, 14, 15, 16, 17], позволяет проводить научные эксперименты для тех ситуаций, где осуществление исследования моделей в реальных условиях сопряжено с трудностями различного характера, и принимать обоснованные решения о жизнеспособности моделей до их практического внедрения.

При этом существует специфика проведения научного экспериментирования моделей, заключающаяся в варьировании отдельными параметрами модели, которые могут влиять на результат ее использования без изменения основных параметров. Такой подход к моделированию имеет безусловное практическое значение, поскольку на сегодняшний день практически не осталось областей, в которых не применялось бы моделирование.

Моделирование применяется в экологии и геофизике, транспортной отрасли, электронике и электротехнике, экономике, строительстве и архитектуре, бизнес-процессах, управлении хозяйствующими субъектами различных уровней, промышленности, политике, деятельности государственных органов различного уровня, медицине и биологии $[2,5]$. В зависимости от направления применения и целей использования моделей варьируются подходы к моделированию. Свою роль в практическом применении моделей играет и прогнозирование поведения моделируемых объектов.

Так, например, глобальная сеть с симуляторами транспортных средств и дисплеями для распределенного моделирования боевых действий в реальном времени на виртуальном поле боя SIMNET, разработанная в США в середине 80-х годов прошлого века, была практически внедрена для обучения, а в последующем для тренировок и поддержки различных военных операций (а также анализе таких операций). В частности, SIMNET применялась в 1992 году в рамках кампании «Буря в пустыне». В качестве еще одного примера практического применения моделей можно назвать внедрение модели объединения центров управления космическими аппаратами, разработанную в рамках программы МКС, в единую распределенную систему (РКК Энергия/Королев ATVCC/Тулуза - NASA JSC/Хьюстон).

В специализированной литературе моделирование дифференцируют на аналитическое, численное и ими- тационное $[3,4,6,8,9]$, каждое из которых служит для решения различных практических задач. В контексте настоящей статьи, направленной на исследование особенностей и значения прогнозирования поведения моделируемых объектов, внимание будет акцентировано на имитационном моделировании, которое лежит в основе проведения научных экспериментов с моделями и по итогам которого может быть принято решение о жизнеспособности модели в практической ситуации.

В наиболее общем понимании имитационное моделирование представляет собой моделирующий алгоритм, который в зависимости от решаемых задач с различной степенью точности воспроизводит функционирование исходной системы. Особенностью имитационного моделирования является возможность воспроизведения поведения моделируемой системы во времени, которое в специализированной литературе принято дифференцировать на физическое, модельное, процессорное [8, 9, 12]. Для целей имитационного моделирования используется понятие модельного времени, и все особенности поведения модели исследуются исходя из заданных значений модельного времени.

Моделируя время эксперимента, направленного на решение задач жизнеспособности модели, например, за счет параллельного выполнения событий, запланированных на разные моменты модельного времени, можно существенно сократить временные затраты на исследование модели. Наиболее отчетливо это прослеживается на распределенной вычислительной системе.

В распределенной модели первичной единицей является логический процесс, который в своем модельном времени выступает в качестве самостоятельной последовательной модели. Путем передачи сообщений логический процесс взаимодействует с другими процессами. В распределенной модели модельное время в разных логических процессах движется с разными скоростями и в некоторый произвольный момент времени оказывается разным. Поэтому для целей правильного воспроизведения последовательности событий в моделируемой системе, необходимо устранить парадоксы времени. Тот процесс продвигает свое время вперед, который получил сообщение от процесса с большшим временем. Если процесс получает сообщение от процесса с меньшим временем, получаем парадокс, т.е. говорят, что процесс, приславший сообщение «из прошлого», отстает во времени $[6,11]$.

Для того, чтобы парадоксов времени в системе не возникало, необходимо предусмотреть специальные программы, которые бы синхронизировали по времени процессы в моделируемой системе. Указанные программы называют также алгоритмами синхронизации мо- 
дельного времени, которые укрупненно дифференцируются на консервативные и оптимистические.

Если синхронизация модельного времени процессов осуществляется под управлением консервативного алгоритма, иными словами, процесс получает сообщения в том же порядке, в котором их посылает ему отправитель. Иначе консервативные алгоритмы блокируют продвижение модельного времени в том случае, если процесс получил сообщение от процесса с меньшим временем.

Оптимистические алгоритмы, напротив, осуществляют откат времени процесса с большим временем до времени процесса, приславшего сообщение, обрабатывают его, а также заново обрабатывают все сообщения от этого времени до текущего в правильной временной последовательности.

Для того, чтобы наглядно представить, как осуществляется моделирование в распределительной системе, проанализируем математические модели консервативного и оптимистического алгоритма. Так, математически модель консервативного алгоритма синхронизации может быть представлена в виде следующего уравнения [3]:

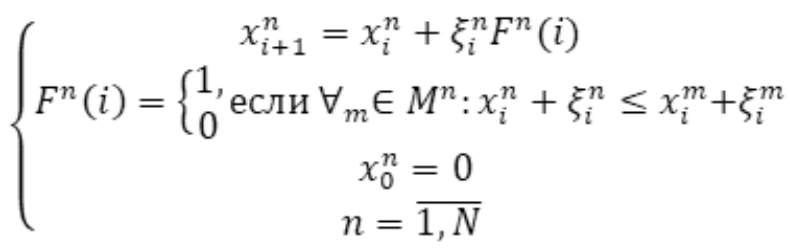

где $x_{i+1}^{n}$ - время процесса $n$ на следующем шаге, $\xi_{i}^{n}$ - локальное приращение времени процесса $n$ (время, затрачиваемое процессом на внутреннюю работу), $n$ - процесс, который продвигает время (получает сообщение от процесса $m), m$ - процесс, посылающий сообщение процессу $n$.

Математически модель оптимистического алгоритма может быть представлена в следующем виде:

$$
\left\{\begin{array}{c}
x_{i+1}^{n}=x_{i}^{n}+\xi_{i}^{n}-\max _{m=\overline{1, N}}\left(\left(x_{i}^{n}-x_{i}^{m}\right) I_{i}^{m \rightarrow n} I_{i}^{x_{1}^{m}<x_{1}^{n}}\right. \\
x_{0}^{n}=0 \\
n=\overline{1, N}
\end{array}\right.
$$

где $I_{i}^{m} \rightarrow n-$ событие, означающее посылку сообщения процессом $m$ процессу $n$,

$I_{i}^{x_{1}^{m}<x_{1}^{n}}$ - событие, означающее, что время процесса $m$ меньше времени процесса.
Если обозначить:

$$
\rho_{i}^{m n}=\left(x_{i}^{n}-x_{i}^{m}\right) I_{i}^{x_{1}^{m}<x_{1}^{n}}, \alpha_{i}^{m n}=I_{i}^{m \rightarrow n},
$$

то получим следующую форму записи оптимистичного алгоритма:

$$
\left\{\begin{array}{c}
x_{i+1}^{n}=x_{i}^{n}+\xi_{i}^{n}-\max _{m=\overline{1, N}}\left(\left(\alpha_{i}^{m n} \rho_{i}^{m n}\right)\right. \\
x_{0}^{n}=0 \\
n=\overline{1, N}
\end{array}\right.
$$

С учетом того, что оценки математических ожиданий величин $\left\{x_{i}^{n}\right\}$, получим следующее выражение:

$$
O^{n}(i)=\left\{\begin{array}{c}
\bar{M}\left(x_{i+1}^{n}\right)=\bar{M}\left(x_{i}^{n}\right)+M\left(\xi_{i}^{n}\right)-\max _{m=\overline{1, N}}\left(\left(\alpha_{i}^{m n} \bar{M}\left(\rho_{i}^{m n}\right)\right.\right. \\
\bar{M}\left(\rho_{i}^{m n}\right)=\left\{\begin{array}{c}
\bar{M}\left(x_{i}^{n}\right)-\bar{M}\left(x_{i}^{m}\right), \text { при } \bar{M}\left(x_{i}^{n}\right)>\bar{M}\left(x_{i}^{m}\right), \\
0, \text { при } \bar{M}\left(x_{i}^{n}\right)>\bar{M}\left(x_{i}^{m}\right), \\
M\left(x_{0}^{n}\right)=0 \\
n=\overline{1, N}
\end{array}\right.
\end{array}\right.
$$

Исходя из вышеприведенных уравнений можно сделать вывод о том, что оба алгоритма синхронизации обладают определенными недостатками. Так, консервативный алгоритм допускает «простои» модельного времени процессов, оптимистический - «откаты» модельного времени.

Для уменьшения количества простоев и откатов модельного времени предлагается алгоритм выбора оптимальной синхронизации процессов, основанный на анализе продвижения модельного времени каждым процессом.

Начало моделирования происходит под управлением консервативного алгоритма. Продвижение времени каждого процесса, участвующего в моделировании, оценивается на предмет изменения приращения его локального времени, а также интенсивности посылки этим процессом сообщений другим процессам, участвующим в моделировании.

Согласно научным исследованиям в области моделирования процессы, участвующие в моделировании, можно условно дифференцировать на три типа. Рассмотрим основные характеристики таких типов $[6,7,8,10$, $12]$.

В рамках первого типа (тип 1) математические ожидания приращений локальных часов имеют небольшой разброс $\leq 50 \%$, процессы активно обмениваются сообщениями $\alpha^{m n} \rightarrow 1$. К указанному типу можно отнести имитационные модели сетей, а также систем обработки сигналов, состоящих из нескольких процессоров и иное коммуникационное оборудование. 


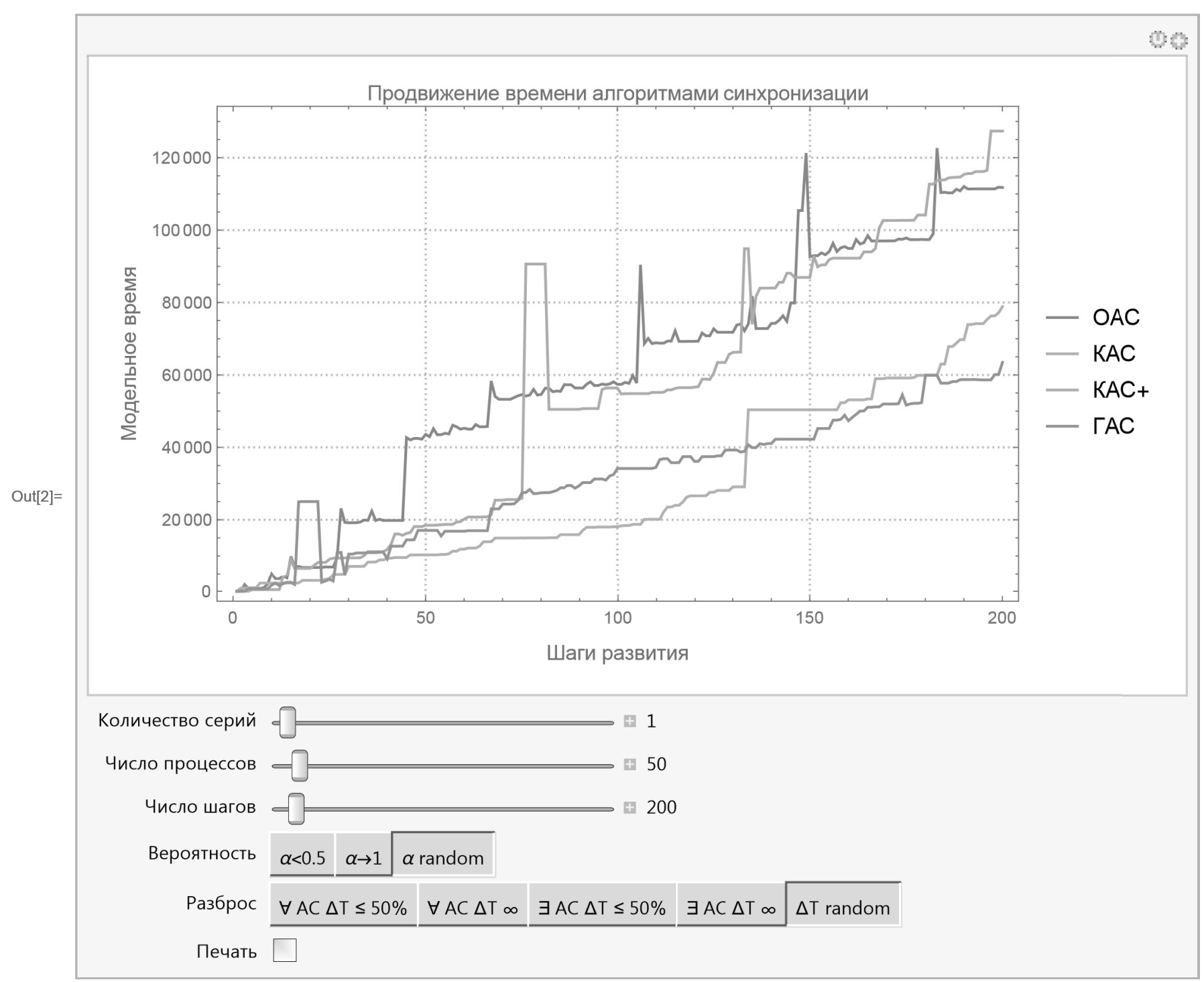

Рис. 1. Соотношение эффективности алгоритмов синхронизации времени в имитационной системе

В рамках процессов второго типа (тип 2) математические ожидания приращений локального времени имеют неограниченный разброс, процессы довольно редко обмениваются сообщениями $a^{m n}<0.5$. Вышеописанному типу процессов соответствуют имитационные модели, в основе которых лежат сложные параллельные алгоритмы, а процессы направлены на осуществление внутренних вычислений, при этом процессы только с входными данными и результатами (например, имитационные модели алгоритмов бортового вычислительного комплекса летательного аппарата).

Третий тип процессов (тип 3) характеризуется ничем не ограниченным разбросом математических ожиданий, поэтому процессы обмениваются сообщениями с разной интенсивностью. К такому типу относят полные модели распределенных вычислительных систем, таких, как имитационная модель бортового навигационного комплекса.

В рамках проведенного в настоящей статье эксперимента, заключающегося в построении математических моделей, было установлено, что в зависимости от распределения приращений локальных часов можно выбирать наиболее эффективный алгоритм синхронизации исходя из того или иного типа процесса, что имеет большое практическое значение. Для того, чтобы описать особенности алгоритма выбора оптимальной синхронизации, представим его в виде пяти последовательных шагов и опишем каждый из этих шагов алгоритма выбоpa.

Шаг 1. Ввод исходных данных: число процессов, участвующих в моделировании, число шагов моделирова- 
ния, вероятность посылки сообщений процессами лежат в интервале от 0 до 1, разброс приращений локальных часов неограничен.

Шаг 2. Старт моделирования. На данном шаге генерируются последовательности посылки сообщений каждым процессом и приращения локального времени для процесса.

Шаг 3. Процессы в соответствии с последовательностями обмениваются сообщениями. Продвижение времени происходит по консервативному алгоритму.

Шаг 4. Если заключительный шаг моделирования не достигнут, то в течение (следующих) 10 шагов анализируются интенсивности посылки сообщений каждым процессом и величины локального приращения времени. Иначе - моделирование заканчивается. Если пара «вероятность посылки сообщений - локальное приращение времени процесса» удовлетворяет какому-либо заданному типу, то следующие 10 шагов процесс осуществляет продвижение времени, синхронизируя его с помощью наиболее оптимального для этой пары алгоритма синхронизации.

Например, процесс посылает сообщения с интенсивностью 6 сообщений за 10 шагов. Это означает, что вероятность посылки составляет 0.6. Если приращение локального времени процесса в результате получения сообщений от других процессов $\otimes 50 \%$, то процесс принадлежит к типу 1. Для данного типа процессов наиболее эффективен консервативный алгоритм синхронизации. Это означает, что следующие 10 шагов процесс будет продвигать свое время с помощью консервативного алгоритма. Если один из параметров процесса указывает на его принадлежность к типу 2, то наиболее оптимальным алгоритмом синхронизации для процесса будет оптимистический и следующие 10 шагов процесс будет продвигать свое время с помощью этого алгоритма.

Шаг 5. Переход на Шаг 4.

Обозначим $\theta^{n}(i)$ функцию, меняющую свое значение в зависимости от типа процесса

$$
\theta^{n}(i)=\left\{\begin{array}{c}
1, \text { если } \alpha_{i}^{m n} \rightarrow 1 ; \Delta T \leq 50 \\
0, \text { если } \alpha^{m n}<0,5 ; \Delta T \rightarrow \infty \\
\text { если } \alpha^{m n} \in[0 . .1] ; \Delta T \rightarrow \in[0 . . \infty] \\
m, n=1, N
\end{array}\right.
$$

Где $\Delta T$, относительное приращение локального времени процессом. Отсюда математическая модель выбора оптимального алгоритма синхронизации, с учетом оценок математического ожидания, будет иметь следующий вид:

$$
\left\{\begin{array}{c}
\bar{M}\left(x_{i+1}^{n}\right)=\overline{K^{n}}(i) \overline{\theta^{n}}(i)+\overline{O^{n}}(i) \overline{\theta^{n}}(i) \\
\overline{K_{i}^{n}}(i)=\bar{M}\left(x_{i}^{n}\right)+M\left(\xi_{i}^{n}\right) \overline{F^{n}}(i) \\
\overline{F^{n}}(i)=\left\{\begin{array}{c}
1, \text { если } \bar{M}\left(x_{i}^{n}\right)+M\left(\xi_{i}^{n}\right) \leq \bar{M}\left(x_{i}^{m}\right)+M\left(\xi_{i}^{m}\right) \\
0 \text { иначе }
\end{array}\right. \\
O^{n}(i)=\bar{M}\left(x_{i+1}^{n}\right)=\bar{M}\left(x_{i}^{n}\right)+M\left(\xi_{i}^{n}\right)-\max _{m=\overline{1, N}}\left(\left(\alpha_{i}^{m n} \bar{M}\left(\rho_{i}^{m n}\right)\right.\right. \\
\bar{M}\left(\rho_{i}^{m n}\right)=\left\{\begin{array}{c}
\bar{M}\left(x_{i}^{n}\right)-\bar{M}\left(x_{i}^{m}\right), \text { при } \bar{M}\left(x_{i}^{n}\right)>\bar{M}\left(x_{i}^{m}\right) \\
0, \text { при } \bar{M}\left(x_{i}^{n}\right)>\bar{M}\left(x_{i}^{m}\right)
\end{array}\right. \\
\theta^{n}(i)\left\{\begin{array}{c}
1, \text { если } \alpha_{i}^{m n} \rightarrow 1 ; \Delta T \leq 50 \\
0, \text { если } \alpha^{m n}<0,5 ; \Delta T \rightarrow \infty \\
\text { если } \alpha^{m n} \in[0 . .1] ; \Delta T \rightarrow \in[0 . . \infty] \\
m, n=\overline{1, N} \\
M(x)_{0}^{n}=0 \\
m, n=\overline{1, N}
\end{array}\right.
\end{array}\right.
$$

Моделирование продвижения времени имитационной модели с помощью данного алгоритма показывает, что эффективность алгоритма возрастает с увеличением числа процессов в моделируемой системе. В этом случае в ряде экспериментов предложенный алгоритм показывает результаты даже лучшие, чем оптимистический и почти в каждом эксперименте лучше, чем консервативный. На рисунке 1 оптимальный алгоритм обозначен как «KAC +».

На практике оптимальный выбор алгоритма позволяет быстрее прийти к выводу о реальной жизнеспособности модели и возможности ее практического применения в зависимости от целей использования модели.

Подводя итог, необходимо отметить, что моделирование как средство проектирования моделей имеет колоссальное практическое и научное значение, поскольку особенности поведение имитационной модели позволяют сделать оценку жизнеспособности модели в реальных условиях еще на этапе проведения эксперимента, что позволяет существенными образом экономить временные и экономические ресурсы. В рамках проведенного в настоящей статье эксперимента доказано, что при построении имитационного процесса функционирования модели путем корректировки (распределения приращений локальных часов) можно выбирать наиболее эффективный алгоритм синхронизации модели в зависимости от типа процесса, участвующего в моделировании. Кроме того, в рамках проведенного исследования разработан и математически описан оптимальный алгоритм моделирования продвижения времени имитационной модели, эффективность которого возрастает с увеличением числа процессов в моделируемой системе. 


\section{ЛИТЕРАТУРА}

1. Богатырев В.А., Богатырев С.В. Резервированная передача данных через агрегированные каналы в сети реального времени // Изв. вузов. Приборостроение.— 2016. — № 9. С. 735-740.

2. Власов М.П., Шимко П.Д. Моделирование экономических процессов.—Ростов-н/Д.: Феникс, 2005.— 409 с.

3. Вознесенская Тамара Васильевна. Исследование эффективности алгоритмов синхронизации времени для систем распределенного имитационного моделирования: дис. ... канд. физ.-мат. наук: 05.13.11/Вознесенская Тамара Васильевна.—М.: МГУ, 2001.— 95 с.

4. Дигрис А.В. Дискретно-событийное моделирование.—-инск: БГУ, 2011. - 175 с.

5. Карпов Ю.Г. Имитационное моделирование систем. Введение в моделирование с AnyLogic 5.— СПб: БХВ-Петербург, 2005.

6. Клейнен Дж. Статистические методы в имитационном моделировании / Пер. с англ.; Под ред. Ю.П. Адлера и В.Н. Варыгина.—М.: Статистика, 1978.

7. К Кобелев Н.Б., Половников В.А., Девятков В.В. Имитационное моделирование.-М.: КУРС. ИНФРА-М, 2015.— 368 с.

8. Колекина А.О., Потекаева Ю.В. Имитационное моделирование: сущность, методы и особенности // APRIORI. Серия: Естественные и технические науки.2016. — № 2.—C. 4-6

9. Кутузов 0.И., Татарникова Т.М. Моделирование систем и сетей телекоммуникаций: Учеб. пособие.—СПб: РГГМУ, 2012.

10. Кутузов 0.И., Татарникова Т.М. К анализу парадигм имитационного моделирования // Научно-технический вестник информационных технологий, механики и оптики.—-2017. - № 3.-С. 552-558.

11. Кутузов 0.И., Татарникова Т.М. Метод ускоренного имитационного моделирования сетевых узлов коммутации // Приборостроение.— 2019. - № 1. С. 23-31.

12. Окольнишников В.В. Представление времени в имитационном моделировании // Вычислительные технологии.— 2005.— № 5.—C. 23.

13. Плютенко А.С. Имитационное моделирование // Вестник МГУП. 2011. № 1. С. 163-170

14. Шеннон Р. Имитационное моделирование систем — искусство и наука. — М.: Издательство «Мир», 1978. — 411 с.

15. https://scholar.google.com.tw/citations?view_op=view_citation\&hl=ru\&user=Xc6gkP8AAAAJ\&citation_for_view=Xc6gkP8AAAAJ: u-x608ySG0sC (дата 06paщения: 10.10.2021).

16. https://scholar.google.com.tw/citations?view_op=view_citation\&hl=ru\&user=Xc6gkP8AAAAJ\&citation_for_view=Xc6gkP8AAAAJ:_FXGoFyzp5QC (дата 06ращения: 10.10.2021).

17. https://scholar.google.com.tw/citations?view_op=view_citation\&hl=ru\&user=Xc6gkP8AAAAJ\&citation_for_view=Xc6gkP8AAAAJ: u5HHmVD_ u08(10.10.2021) (дата обращения: 10.10.2021).

○ Пантюхов Дмитрий Валерьевич ( gospamme@ya.ru ).

Журнал «Современная наука: актуальные проблемы теории и практики» 